Check for updates

Cite this: RSC Adv., 2017, 7, 44961

Received 21st August 2017

Accepted 12th September 2017

DOI: $10.1039 / c 7 r a 09229 f$

rsc.li/rsc-advances

\section{Network-based collaborative filtering recommendation model for inferring novel disease- related miRNAs}

\author{
Changlong Gu, (D) ${ }^{a}$ Bo Liao, ${ }^{* a}$ Xiaoying Li, ${ }^{a}$ Lijun Cai, ${ }^{a}$ Haowen Chen, ${ }^{a}$ Keqin Li ${ }^{b}$ \\ and Jialiang Yang ${ }^{\mathrm{C}}$
}

MicroRNAs (miRNAs) play important roles in the pathogenesis and development of many complex diseases. The experimental confirmation of disease-related miRNAs is costly and time-consuming. An efficient and accurate computational model for identifying potential miRNA-disease associations is a useful supplement for experimental approaches. In this study, we develop a new method for measuring miRNA and disease similarities, which are key issues in identifying miRNA-disease associations, based on normalized mutual information. Subsequently, a network-based collaborative filtering recommendation model, network-based collaborative filtering (NetCF), is proposed for predicting potential miRNAdisease associations by integrating miRNA and disease similarities along with experimentally verified miRNA-disease associations. Leave-one-out cross validation is implemented to evaluate the predicted performance of NetCF. NetCF obtains a reliable AUC value of 0.8960 , which is superior to other competitive methods. Implementing NetCF to predict lung cancer and prostate cancer-related miRNAs, $94 \%$ of the top 50 predicted miRNAs of each cancer have been confirmed by other databases.

\section{Introduction}

MicroRNAs (miRNAs) are small non-coding RNA molecules $(\sim 22 \mathrm{nt})$ that can regulate gene expression at the posttranscriptional level. ${ }^{\mathbf{1}}$ Accumulating evidence indicates that miRNA mutations and dysregulations are closely related to various human diseases, ${ }^{2}$ including cardiovascular diseases, ${ }^{3}$ schizophrenia, ${ }^{4}$ and cancer., ${ }^{5,6}$ Thus, identification of diseaserelated miRNAs will be helpful in the diagnosis, treatment, and prevention of diseases. Biological experiment methods such as microarray profiling and qRT-PCR can effectively identify miRNA-disease associations. For instance, Manavalan et $a .^{7}$ confirmed miRs-10a, 21, 22, 29a, 93, 125b, 181, 200a, 200b, 200c, 205, and 222, which are associated with human breast cancer cells by microarray profiling. Some associations between miRNAs and diseases have been reported over the last few years. A number of researchers have constructed a miRNAdisease association database by collecting data from experiments that support human miRNAs and disease associations from published studies. These databases include HMDD, ${ }^{8}$ miR2Disease, ${ }^{9}$ dbDEMC, ${ }^{\mathbf{1 0 , 1 1}}$ and PhenomiR, ${ }^{12}$ which provide

${ }^{a}$ College of Information Science and Engineering, Hunan University, Changsha, Hunan 410082, China.E-mail: dragonbw@163.com

${ }^{b}$ Department of Computer Science, State University of New York, New Paltz, New York 12561, USA

${ }^{c}$ Department of Genetics and Gnomic Science, Icahn School of Medicine at Mount Sinai, New York 10029, USA a strong data basis for studying miRNAs. However, only a small amount of miRNA-disease associations have been reported in these databases. Due to the high cost and long time required for the identification of disease-related miRNAs through biological experimental methods, effective computational approaches for predicting disease-related miRNAs received great attention.

Several computational models have been developed to infer potential miRNA-disease associations. The aforementioned methods can be divided into two categories: network-based methods and machine-learning-based methods. ${ }^{13}$

The key problem of network-based methods is predicting that miRNA-disease associations are similar to the calculation among miRNAs and diseases over the networks. Some approaches have been reported to measure miRNA and disease similarities; ${ }^{\mathbf{1 4 - 1 6}}$ Zou et al. ${ }^{\mathbf{1 7}}$ reviewed the main similarity computation methods. Based on the common assumption that miRNAs are normally associated with phenotypically similar diseases and vice versa, Jiang et al. ${ }^{18}$ constructed a functionally related miRNA network and a human phenome-miRNAome network to prioritize potential disease-related miRNAs. However, the main limitation of this method is the high number of false positives that are produced in the miRNA target prediction step. To improve predicted performance, Jiang et $a{ }^{19}{ }^{19}$ subsequently proposed a Naive Bayes model to infer disease-related miRNAs by integrating multiple types of data resources. Some researchers have successfully applied the random walk algorithm to predict miRNA-disease associations. ${ }^{20-22}$ Based on global network similarity measures, Chen 
et $a l .{ }^{21}$ constructed a miRNA-miRNA functional similarity network and implemented Random Walk with Restart (RWR) from known disease-related miRNA seed nodes to prioritize potential disease-related miRNAs. By integrating disease-gene associations, miRNA-mRNA interactions, and protein-protein interactions, Shi et al. $^{22}$ developed an improved method based on RWR to predict disease-related miRNAs and achieved a satisfactory performance with cross validation. Liu et al. ${ }^{\mathbf{2 0}}$ recently constructed a heterogeneous network by connecting disease and miRNA similarity subnetworks using known miRNA-disease associations and extended RWR method to infer potential miRNA-disease associations in the heterogeneous network. Although the RWR method presented good performance in predicting miRNA-disease associations, it cannot be implemented to diseases without any known associated miRNA. A similarity-based method called networkconsistency-based inference (NetCBI) has been proposed by Chen et $a l .{ }^{23}$ to predict miRNA-disease associations. NetCBI can predict disease-related miRNAs when diseases have no known associated miRNA. However, the cross validation exhibited poor performance. Xuan et al. ${ }^{24}$ presented a novel method (HDMP) after considering the local information of network, based on weighted $k$ where most similar neighbors predict diseaserelated miRNAs. Cross validation and case studies of HDMP indicate good predicted performance, but it does not work for diseases without known related miRNAs. Furthermore, based solely on gene expression profiles, Zhao et al. ${ }^{25}$ presented a computational framework to identify the cancer-related miRNAs, and constructed a cancer-miRNA-pathway network, which can help explain how miRNAs are involved in cancer. Recently, Qin et al. ${ }^{26}$ proposed a method to predict diseaseassociated miRNAs based on protein domains. The results on real datasets demonstrate the effectiveness of the approach.

Some researchers proposed machine-learning-based methods to predict potential miRNA-disease associations. To distinguish positive miRNA-disease from large-scale negative miRNA-disease associations, Jiang et al. ${ }^{27}$ extracted a set of features from each positive and negative microRNAdisease association and trained a Support Vector Machine (SVM) classifier to predict novel miRNA-disease associations. Based on miRNA-disease heterogeneous network, Zeng et al. ${ }^{28}$ used a path-based measure named HeteSim, ${ }^{29}$ to calculate relevance between objects in the heterogeneous network and combined HeteSim scores with a machine learning method to predict novel miRNA-disease associations. The challenge of using machine-learning-based methods for predicting novel miRNA-disease associations is the difficulty in obtaining negative samples (a miRNA is not associated with a disease). Given that limited trials do not provide enough evidence to prove that miRNA is not associated with a disease, Chen et al. $^{30}$ proposed the Regularized Least Squares for miRNAdisease Associations (RLSMDA) to prioritize potential miRNA-disease associations without utilizing negative samples. RLSMDA is a semi-supervised classification algorithm that can predict associations for disease without any associated miRNA.
By analyzing the aforementioned methods, the existing computation methods for predicting miRNA-disease associations are restricted by several limitations. First, some methods ${ }^{\mathbf{1 8}}$ calculated miRNA similarities based on miRNA-mRNA database, and would produce higher false positives in the miRNA target prediction step. Second, some approaches ${ }^{21,23,24,30}$ calculated miRNA similarity based on the known miRNA-disease associations and evaluated their predicted performance through leave-one-out cross validation (LOOCV). The predicted performance would be overestimated, given that the similarity calculation has included the removal of the miRNA-disease association when LOOCV is performed. ${ }^{13}$ Third, some methods ${ }^{21,24}$ cannot be implemented to disease without any known associated miRNA. Finally, some machine-learningbased methods ${ }^{27,28}$ require negative samples to train classifiers; however, obtaining the negative samples is difficult.

To solve these complications, a network-based collaborative filtering recommend algorithm (NetCF) is proposed to reveal the potential associations between miRNAs and diseases. NetCF integrates miRNA and disease similarities along with the known miRNA-disease associations to reveal potential miRNA-disease associations. NetCF exhibits a clear advantage over other methods, which involve various features, such as LOOCV, case studies, global prediction for all diseases, prediction of disease without any known related miRNA (isolated disease), and prediction of miRNA with no associated disease (novel miRNA).

The main contributions of the paper are summarized as follows.

(1) miRNA similarities are calculated by experimentally verifying miRNA-mRNA interactions to eliminate false positives of the miRNA-target.

(2) miRNA and disease similarities are not dependent on the known miRNA-disease associations, so that LOOCV will not be too high to estimate its predicted performance.

(3) NetCF integrates miRNA- and disease-similarity-based recommendations to predict potential miRNA-disease associations. Therefore, when a disease is not related with any miRNA, the association can be predicted by miRNA-similarity-based recommendation. For the same reason, NetCF can also be applied to predict novel miRNA.

(4) NetCF uses similarity information and known miRNAdisease associations to infer potential miRNA-disease associations without requiring negative sample information.

\section{Materials and methods}

The flowchart of NetCF and the calculation of the predictive score between miRNA $i$ and disease $j$ are shown in Fig. 1.

\subsection{Human miRNA-disease associations}

The experimentally verified human miRNA-disease associations are downloaded from last updated HMDD database. ${ }^{8}$ We perform the following operations on these associations. The disease names are mapped to the disease $\mathrm{MeSH}$ description (https:/www.ncbi.nlm.nih.gov/mesh). Repeated associations and several diseases without any MeSH descriptors or tree 
(1) miRNA similarity measurement

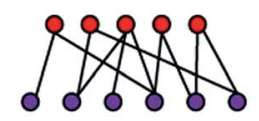

miRNA-mRNA interactions
$\Rightarrow$

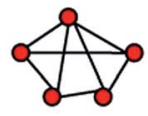

miRNA functional similarity (MM)
- miRNA

- Disease

- mRNA

(2) Disease similarity measurement

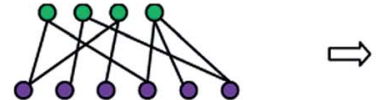

Disease-mRNA interactions

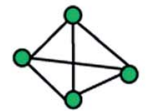

Disease functional similarity (DF)

\begin{tabular}{|c|c|}
\hline Disease & c \\
\hline Neoplasms & $\cos$ \\
\hline Neoplasms by site & cos. 588 \\
\hline Breast Neoplasms & cos. $588.180 ;$ c 17.800 .090 .500 \\
\hline Lung Neoplasms & CO4.588.894.797.520; C08.381 \\
\hline
\end{tabular}

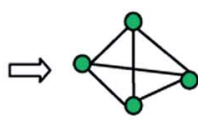

\begin{tabular}{l|l}
\hline Lung Neoplasms & C04.588.894.797.520; C08.381.540; C08.785.520 \\
\hline
\end{tabular}

MeSH tree structure

Disease semantic similarity $(D S)$

(3) Collaborative filtering algorithm

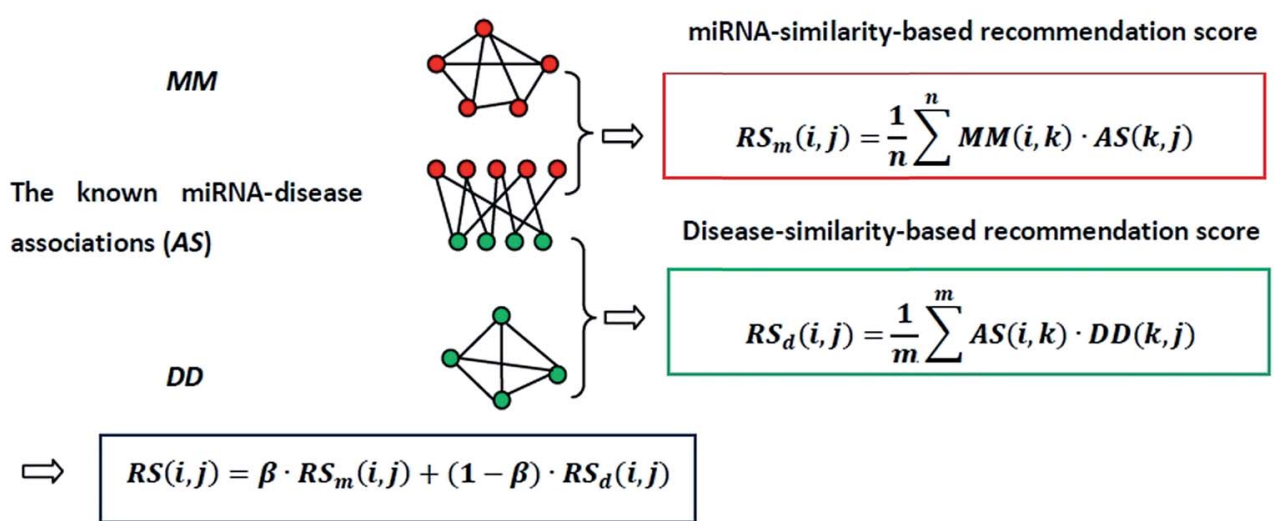

Fig. 1 The flowchart of NetCF. NetCF calculates the association score between miRNA $i$ and disease $j$ is divided into three steps: (1) miRNA similarities are calculated based on known miRNA-mRNA interactions. (2) The disease functional similarities are calculated based on the known disease-mRNA interactions, and the disease semantic similarities are calculated based on disease MeSH tree structures, and then they are integrated to obtain disease similarities. (3) Based on the similarity information between miRNA $i$ and their neighbors and the association information between the neighbors and disease $j$, the miRNA-similarity-based recommendation score between miRNA $i$ and disease $j$ is calculated. Using a similar method to calculate the disease-similarity-based recommendation score, and then they were integrated to obtain the final prediction score.

numbers are removed. Moreover, the experimentally verified disease-related genes from the DisGeNET database ${ }^{31}$ are used to calculate disease similarity; thus, the disease names from the DisGeNET database are also mapped to the MeSH description and the diseases not found in the DisGeNET database are removed. After this treatment, 5048 distinct high-quality experimentally verified miRNA-disease associations are obtained, including 475 miRNAs and 334 diseases. We use this dataset as the benchmark dataset and variables $\mathrm{nm}$ and nd to represent the number of miRNAs and diseases, respectively. The adjacency matrix of miRNA-disease associations is denoted by matrix AS, whereas the entity $\operatorname{AS}(i, j)$ in row $i$ and column $j$ is 1 if miRNA $i$ is associated with disease $j$, and 0 otherwise.

\section{2. miRNA similarity measurement}

The miRNA and disease similarities calculated based on known miRNA-disease associations overestimate predicted performance when cross validation is implemented. Based on the assumption that miRNAs with similar functions tend to be associated with similar target genes (mRNA), we presented the measurement of the functional similarity of two miRNAs by considering their related target genes. To eliminate false positive miRNA-target, miRNA similarities are calculated using experimentally verified miRNA-mRNA interactions downloaded from the miRTarBase database, ${ }^{32,33}$ Release 6.1. Let set $T_{\mathrm{m}}{ }^{A}=\left\{T_{\mathrm{m}}{ }^{A}(1), T_{\mathrm{m}}{ }^{A}(2), \ldots, T_{\mathrm{m}}{ }^{A}(m a)\right\}$ and set $T_{\mathrm{m}}{ }^{B}=$ 
$\left\{T_{\mathrm{m}}{ }^{B}(1), T_{\mathrm{m}}{ }^{B}(2), \ldots, T_{\mathrm{m}}{ }^{B}(\mathrm{mb})\right\}$ denote the target gene sets of miRNAs $A$ and $B$, where variables $m a$ and $m b$ are the number of target genes of miRNA $A$ and $B$, respectively. The information entropy of $T_{\mathrm{m}}{ }^{A}$ is defined in eqn (1):

$$
\begin{aligned}
& \left\{H\left(T_{\mathrm{m}}{ }^{A}\right)=-\sum_{i=1}^{m a} p\left(T_{\mathrm{m}}{ }^{A}(i)\right) \log _{2}\left(p\left(T_{\mathrm{m}}{ }^{A}(i)\right)\right)\right. \\
& p\left(T_{\mathrm{m}}{ }^{A}(i)\right)=\frac{n\left(T_{\mathrm{m}}{ }^{A}(i)\right)}{N}
\end{aligned}
$$

where $N$ is the number of miRNA-mRNA interactions; $n\left(T_{\mathrm{m}}{ }^{A}(i)\right)$ is the number of the $i^{\text {th }}$ target gene of miRNA $A$ in the miRNA-mRNA set; $p\left(T_{\mathrm{m}}{ }^{A}(i)\right)$ is the frequency of the $i^{\text {th }}$ target gene of miRNA $A$ in the miRNA-mRNA set; and $H\left(T_{\mathrm{m}}{ }^{A}\right)$ is the information entropy of $T_{\mathrm{m}}{ }^{A}$.

The normalized mutual information (NMI) of $T_{\mathrm{m}}{ }^{A}$ and $T_{\mathrm{m}}{ }^{B}$ is used to measure the functional similarity of miRNAs $A$ and $B$ :

$$
\operatorname{MM}(A, B)=\frac{2 H\left(T_{\mathrm{m}}{ }^{A} \cap T_{\mathrm{m}}{ }^{B}\right)}{H\left(T_{\mathrm{m}}{ }^{A}\right)+H\left(T_{\mathrm{m}}{ }^{B}\right)}
$$

where $H\left(T_{\mathrm{m}}{ }^{A}\right), H\left(T_{\mathrm{m}}{ }^{B}\right)$ and $H\left(T_{\mathrm{m}}{ }^{A} \cap T_{\mathrm{m}}{ }^{B}\right)$ represent the information entropy of $T_{\mathrm{m}}{ }^{A}, T_{\mathrm{m}}{ }^{B}$ and the intersection set of $T_{\mathrm{m}}{ }^{A}$ and $T_{\mathrm{m}}{ }^{B}$, respectively. The functional similarity between two miRNAs is measured by eqn (2) according to their common target genes and the information entropy of their respective target gene sets; and it is standardized based on NMI. Matrix MM is the functional similarity matrix, and $\operatorname{MM}(i, j)$ in row $i$ and column $j$ represents the similarity between miRNAs $i$ and $j$.

\subsection{Disease similarity measurement}

Disease similarity is composed of two parts: disease functional and disease semantic similarities.

Based on the common assumption that two diseases with similar functions are normally associated with similar target genes, we also used the NMI of two disease-target gene sets to measure their functional similarity. Experimentally-verified disease-mRNA interactions are employed in this study and downloaded from the DisGeNET database. We use sets $T_{\mathrm{d}}{ }^{A}=$ $\left\{T_{\mathrm{d}}{ }^{A}(1), T_{\mathrm{d}}{ }^{A}(2), \ldots, T_{\mathrm{d}}{ }^{A}(d a)\right\}$ and $T_{\mathrm{d}}{ }^{B}=\left\{T_{\mathrm{d}}{ }^{B}(1), T_{\mathrm{d}}{ }^{B}(2), \ldots, T_{\mathrm{d}}{ }^{B}(d b)\right\}$ to denote the target gene set of diseases $A$ and $B$, where $d a$ and $d b$ refer to the number of target genes of diseases $A$ and $B$, respectively. Similar to the miRNA function similarity calculation, the NMI of $T_{\mathrm{d}}{ }^{A}$ and $T_{\mathrm{d}}{ }^{B}$ is used to measure the functional similarity between diseases $A$ and $B$ as follows:

$$
\mathrm{DF}(A, B)=\frac{2 H\left(T_{\mathrm{d}}{ }^{A} \cap T_{\mathrm{d}}{ }^{B}\right)}{H\left(T_{\mathrm{d}}{ }^{A}\right)+H\left(T_{\mathrm{d}}{ }^{B}\right)}
$$

where $H\left(T_{\mathrm{d}}{ }^{A}\right), H\left(T_{\mathrm{d}}{ }^{B}\right)$ and $H\left(T_{\mathrm{d}}{ }^{A} \cap T_{\mathrm{d}}{ }^{B}\right)$ represent the information entropy of $T_{\mathrm{d}}{ }^{A}, T_{\mathrm{d}}{ }^{B}$ and the intersection set of $T_{\mathrm{d}}{ }^{A}$ and $T_{\mathrm{d}}{ }^{B}$, respectively. The functional similarity between two diseases is determined by their common target genes and the information entropy of their respective target gene sets.

An improved form of Wang's method ${ }^{14}$ for disease semantic similarity calculation is implemented in this paper. This method calculates disease semantic similarity based on the hierarchical structure of MeSH. A disease can be described as a directed acyclic graph (DAG), in which the nodes represent diseases, whereas the links represent the relationship between nodes. Let
$\mathrm{DAG}_{\mathrm{d}}=\left(d, T_{\mathrm{d}}, E_{\mathrm{d}}\right)$ denote the DAG graph of disease $d$, where $T_{\mathrm{d}}$ is the node set (all ancestor nodes of disease $d$ including disease $d$ itself) and $E_{\mathrm{d}}$ is the connected edge set. Wang's method defines the semantic contribution of node $t \in T_{\mathrm{d}}$ as follows:

$$
D_{\mathrm{d}}(t)=\left\{\begin{array}{l}
1 \quad \text { if } t=d \\
\max \left\{\Delta \times D_{\mathrm{d}}\left(t^{\prime}\right) \mid t^{\prime} \in \text { children of } t\right\} \quad \text { if } t \neq d
\end{array}\right.
$$

where $\Delta$ is the semantic contribution factor, and $\Delta=0.5$ is used in their experiments. In this manner, the semantic contribution of the disease term is defined by only considering the hierarchical structure of the DAG graph. For example, the DAG graph of "Breast Neoplasms" and the semantic contribution (sc) values of each node are shown in Fig. 2.

The importance of the disease term itself is considered; for example, disease terms "liver neoplasms" and "neoplasms" are specifically described as "liver neoplasms", such that its semantic contribution value should be greater than the contribution value of "neoplasms." We use information content (IC) to measure the importance of the disease term itself:

$$
\mathrm{IC}(t)=-\log _{2}(p(t))
$$

where $p(t)$ is the frequency of disease term $t$ in the MeSH disease term set. Evidently, the larger the $p(t)$ is, the more common description of disease $t$ is and the smaller the semantic contribution value of disease $t$ is. In this paper, the $\mathrm{MeSH}$ disease term set is downloaded from the MeSH homepage (2017 MeSH files), which contains 57840 disease tree numbers with their corresponding disease terms. The semantic contribution value of disease $t$ is obtained by integrating $D_{\mathrm{d}}(t)$ and $\mathrm{IC}(t)$ as $C_{\mathrm{d}}(t)=D_{\mathrm{d}}(t) \times \mathrm{IC}(t)$. The semantic similarity of diseases $A$ and $B$ can be calculated by their shared ancestors as follows:

$$
\operatorname{DS}(A, B)=\frac{\sum_{t \in\left(T_{A} \cap T_{B}\right)}\left(C_{A}(t)+C_{B}(t)\right)}{\sum_{t \in T_{A}}\left(C_{A}(t)\right)+\sum_{t \in T_{B}}\left(C_{B}(t)\right)}
$$

Finally, the similarity between diseases $A$ and $B$ is calculated by integrating disease functional and disease semantic similarities as follows:

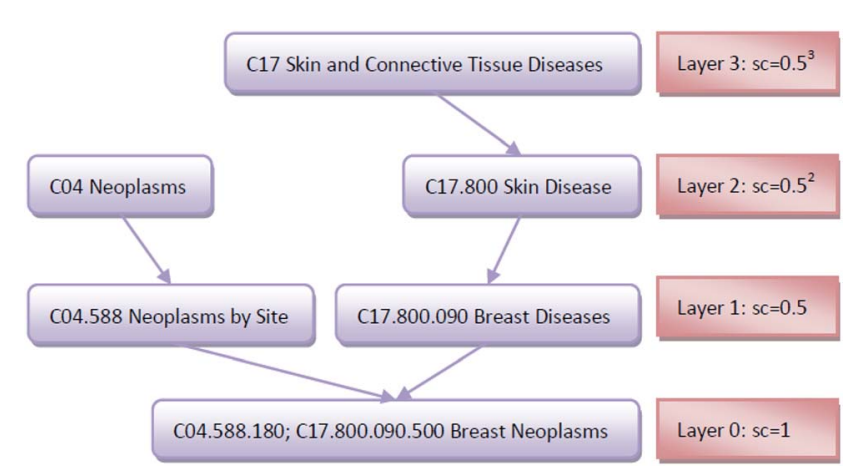

Fig. 2 The DAG graph of "Breast Neoplasms" and the semantic contribution values of each node. 


$$
\mathrm{DD}(A, B)=\alpha \mathrm{DF}(A, B)+(1-\alpha) \operatorname{DS}(A, B)
$$

where $\alpha \in[0,1]$ is the weight coefficient used to indicate the importance of disease functional and semantic similarities. In our experiments, we regard the two parts are equally important; thus, $\alpha$ set to 0.5 . Matrix DD is the similarity matrix and $\mathrm{DD}(i, j)$ in row $i$ and column $j$ represents the similarity between diseases $i$ and $j$.

\subsection{NetCF}

Collaborative filtering (CF) algorithm is one of the most important techniques used by recommender systems, ${ }^{34}$ and it has enjoyed tremendous success in e-business, marketing, and other personalized recommendation services. CF algorithm generates recommendations or predictions based on observed preferences. ${ }^{35}$ In this study, by integrating the miRNA similarity, disease similarity, and known miRNAdisease association networks, a NetCF recommendation model is proposed to predict potential miRNA-disease associations. NetCF is implemented in three steps as follows: (1) miRNA-similarity-based recommendation score calculation; (2) disease-similarity-based recommendation score calculation; (3) and the calculation of final predictor score of potential miRNA-disease associations by integrating the results of steps (1) and (2).

The detailed implementation procedure of NetCF for calculating the predictor score between miRNA $i$ and disease $j$ is as follows.

First, based on the similarity information between miRNA $i$ and their neighbors and the association information between the neighbors and disease $j$, the miRNA-similarity-based recommendation score between miRNA $i$ and disease $j$ is calculated. Evidently, if the similarity between a neighbor and miRNA $i$ is extremely small, then the contribution of the neighbor can be ignored. The miRNA-similarity-based recommendation score according to the $n$ most similar neighbors of miRNA $i$ is calculated as follows:

$$
\mathrm{RS}_{\mathrm{m}}(i, j)=\frac{1}{n} \sum_{k=1}^{n} \mathrm{MM}(i, k) \times \operatorname{AS}(k, j)
$$

where $\operatorname{MM}(i, k)$ is the similarity value between the $k^{\text {th }}$ most similar neighbor of miRNA $i$ and miRNA $k$, and AS $(k, j)$ is the association information between the $k^{\text {th }}$ most similar neighbor of miRNA $i$ and disease $j$. In our experiments, the value of parameter $n$ is $10 \%$ of the miRNA number $(\mathrm{nm} / 10)$. Matrix $\mathrm{RS}_{\mathrm{m}}$ is the miRNA-similarity-based recommendation score matrix, and $\operatorname{RS}_{\mathrm{m}}(i, j)$ in row $i$ and column $j$ represents the miRNAsimilarity-based recommendation score between miRNA $i$ and disease $j$.

Second, the disease-similarity-based recommendation score between miRNA $i$ and disease $j$ is calculated based on the similarity information between disease $j$ and their neighbors and the association information between the neighbors and miRNA $i$. For the same reason, the disease-similarity-based recommendation score is calculated by the $m$ most similar neighbors of disease $j$ as follows:

$$
\mathrm{RS}_{\mathrm{d}}(i, j)=\frac{1}{m} \sum_{k=1}^{m} \mathrm{AS}(i, k) \times \mathrm{DD}(k, j)
$$

where $\mathrm{DD}(k, j)$ is the similarity value between the $k^{\text {th }}$ most similar neighbor of disease $k$ and disease $j$, and $\operatorname{AS}(i, k)$ is the association information between the $k^{\text {th }}$ most similar neighbor of disease $j$ and miRNA $i$. In our experiments, the value of parameter $m$ is $10 \%$ of the disease number (nd/10). Matrix $\mathrm{RS}_{\mathrm{d}}$ is the diseasesimilarity-based recommendation score matrix, and $\mathrm{RS}_{\mathrm{d}}(i, j)$ in row $i$ and column $j$ represents the disease-similarity-based recommendation score between miRNA $i$ and disease $j$.

Finally, miRNA- and disease-similarity-based recommendation scores are integrated as the final recommendation score of miRNA $i$ and disease $j$ as follows:

$$
\operatorname{RS}(i, j)=\beta \operatorname{RS}_{\mathrm{m}}(i, j)+(1-\beta) \mathrm{RS}_{\mathrm{d}}(i, j)
$$

where $\mathrm{RS}_{\mathrm{m}}\left(i_{j}\right)$ and $\mathrm{RS}_{\mathrm{d}}\left(i_{j}\right)$ are the miRNA-based and the disease-similarity-based recommendation scores between

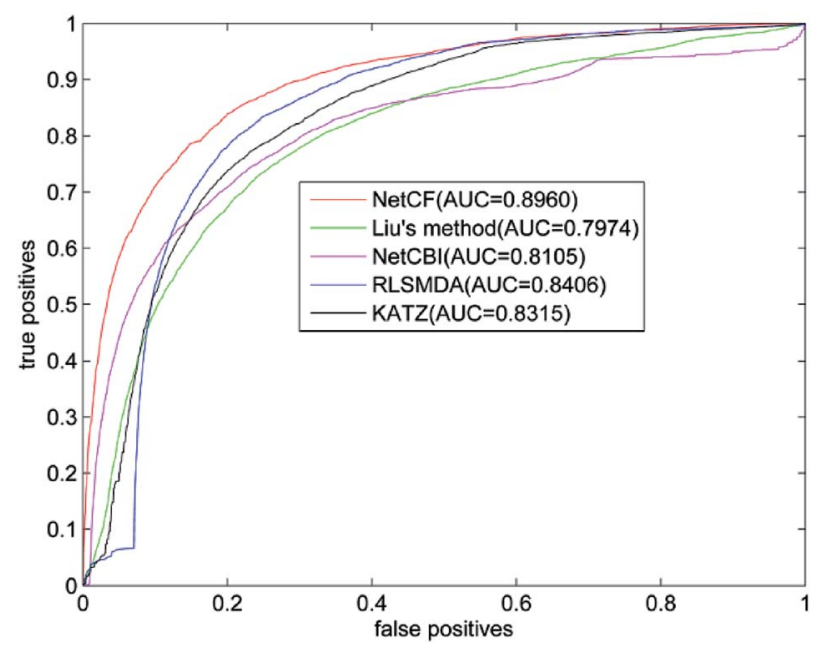

Fig. 3 Performance comparisons of NetCF, Liu's method, NetCBI, RLSMDA, and KATZMDA in terms of ROC curves and AUCs based on LOOCV.

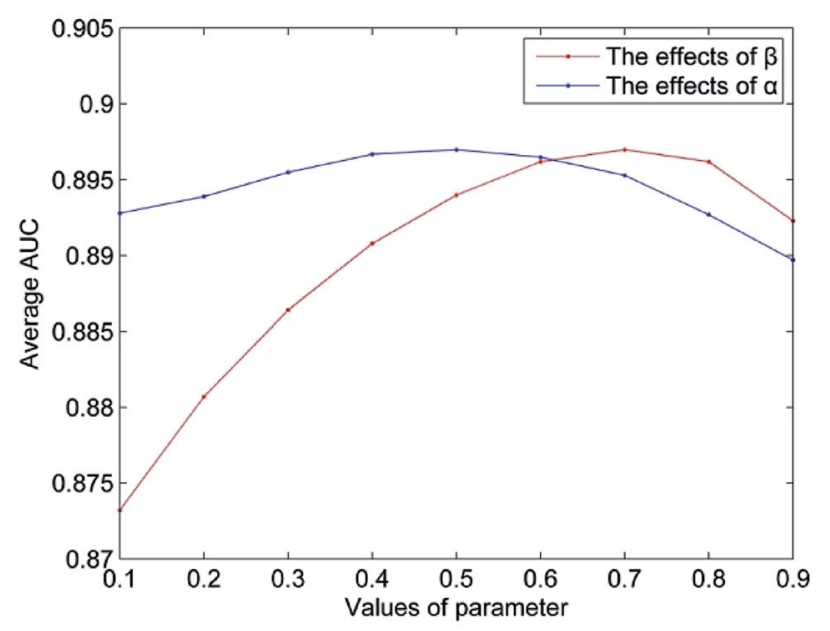

Fig. 4 The parameters effects on NetCF. 
Table 1 The top 50 potential lung cancer-related miRNAs predicted by NetCF, 47 of them were confirmed by dbDEMC and PhenomiR databases

\begin{tabular}{|c|c|c|}
\hline Rank & miRNA & Evidences \\
\hline 1 & hsa-mir-16 & $\begin{array}{l}\text { dbDEMC, } \\
\text { PhenomiR }\end{array}$ \\
\hline 2 & hsa-mir-195 & $\begin{array}{l}\text { dbDEMC, } \\
\text { PhenomiR }\end{array}$ \\
\hline 3 & hsa-mir-429 & dbDEMC \\
\hline 4 & hsa-mir-15a & $\begin{array}{l}\text { dbDEMC, } \\
\text { PhenomiR }\end{array}$ \\
\hline 5 & hsa-mir-451a & dbDEMC \\
\hline 6 & hsa-mir-141 & $\begin{array}{l}\text { dbDEMC, } \\
\text { PhenomiR }\end{array}$ \\
\hline 7 & hsa-mir-106b & $\begin{array}{l}\text { dbDEMC, } \\
\text { PhenomiR }\end{array}$ \\
\hline 8 & hsa-mir-449a & PhenomiR \\
\hline 9 & hsa-mir-193b & $\begin{array}{l}\text { dbDEMC, } \\
\text { PhenomiR }\end{array}$ \\
\hline 10 & hsa-mir-302d & PhenomiR \\
\hline 11 & hsa-mir-383 & PhenomiR \\
\hline 12 & hsa-mir-20b & $\begin{array}{l}\text { dbDEMC, } \\
\text { PhenomiR }\end{array}$ \\
\hline 13 & hsa-mir-194 & $\begin{array}{l}\text { dbDEMC, } \\
\text { PhenomiR }\end{array}$ \\
\hline 14 & hsa-mir-130a & $\begin{array}{l}\text { dbDEMC, } \\
\text { PhenomiR }\end{array}$ \\
\hline 15 & hsa-mir-151a & dbDEMC \\
\hline 16 & hsa-mir-99a & $\begin{array}{l}\text { dbDEMC, } \\
\text { PhenomiR }\end{array}$ \\
\hline 17 & hsa-mir-296 & $\begin{array}{l}\text { dbDEMC, } \\
\text { PhenomiR }\end{array}$ \\
\hline 18 & hsa-mir-320a & $\begin{array}{l}\text { dbDEMC, } \\
\text { PhenomiR }\end{array}$ \\
\hline 19 & hsa-mir-215 & PhenomiR \\
\hline 20 & hsa-mir-378a & dbDEMC \\
\hline 21 & hsa-mir-15b & $\begin{array}{l}\text { dbDEMC, } \\
\text { PhenomiR }\end{array}$ \\
\hline 22 & hsa-mir-153 & $\begin{array}{l}\text { dbDEMC, } \\
\text { PhenomiR }\end{array}$ \\
\hline 23 & hsa-mir-328 & $\begin{array}{l}\text { dbDEMC, } \\
\text { PhenomiR }\end{array}$ \\
\hline 24 & hsa-mir-149 & $\begin{array}{l}\text { dbDEMC, } \\
\text { PhenomiR }\end{array}$ \\
\hline 25 & hsa-mir-302c & PhenomiR \\
\hline 26 & hsa-mir-130b & $\begin{array}{l}\text { dbDEMC, } \\
\text { PhenomiR }\end{array}$ \\
\hline 27 & hsa-mir-122 & PhenomiR \\
\hline 28 & hsa-mir-302a & PhenomiR \\
\hline 29 & hsa-mir-449b & PhenomiR \\
\hline 30 & hsa-mir-10a & $\begin{array}{l}\text { dbDEMC, } \\
\text { PhenomiR }\end{array}$ \\
\hline 31 & hsa-mir-152 & $\begin{array}{l}\text { dbDEMC, } \\
\text { PhenomiR }\end{array}$ \\
\hline 32 & hsa-mir-147 & dbDEMC \\
\hline 33 & hsa-mir-302b & PhenomiR \\
\hline 34 & hsa-mir-204 & $\begin{array}{l}\text { dbDEMC, } \\
\text { PhenomiR }\end{array}$ \\
\hline 35 & hsa-mir-181d & $\begin{array}{l}\text { dbDEMC, } \\
\text { PhenomiR }\end{array}$ \\
\hline 36 & hsa-mir-139 & $\begin{array}{l}\text { dbDEMC, } \\
\text { PhenomiR }\end{array}$ \\
\hline 37 & hsa-mir-372 & PhenomiR \\
\hline 38 & hsa-mir-196b & $\begin{array}{l}\text { dbDEMC, } \\
\text { PhenomiR }\end{array}$ \\
\hline
\end{tabular}

Table 1 (Contd.)

\begin{tabular}{lll}
\hline Rank & miRNA & Evidences \\
\hline 39 & hsa-mir-423 & $\begin{array}{l}\text { dbDEMC, } \\
\text { PhenomiR } \\
\text { dbDEMC, } \\
\text { PhenomiR }\end{array}$ \\
40 & hsa-mir-148b & Unconfirmed \\
& hsa-mir-520g & dbDEMC \\
41 & hsa-mir-615 & PhenomiR \\
42 & hsa-mir-151b & dbDEMC, \\
43 & hsa-mir-373 & PhenomiR \\
44 & hsa-mir-452 & PhenomiR \\
45 & hsa-mir-367 & Unconfirmed \\
& hsa-mir-630 & dbDEMC, \\
46 & hsa-mir-324 & PhenomiR \\
47 & hsa-mir-519c & Unconfirmed \\
48 & hsa-mir-625 & dbDEMC \\
49 & &
\end{tabular}

miRNA $i$ and disease $j$, respectively. Parameter $\beta \in[0,1]$ is the integrated parameter used to indicate the importance of $\mathrm{RS}_{\mathrm{m}}(i, j)$ and $\mathrm{RS}_{\mathrm{d}}(i, j)$. In our experiments, parameter $\beta$ is set to $\beta=\mathrm{nm} /$ $(\mathrm{nm}+\mathrm{nd})$ balance the numbers of miRNAs and diseases. Matrix RS is the final recommendation score matrix, and $\operatorname{RS}(i, j)$ in row $i$ and column $j$ represents the final recommendation score between miRNA $i$ and disease $j$.

\section{Results}

\subsection{Performance evaluation}

The predicted performance of NetCF is compared with the other four state-of-the-art computational models, i.e., Liu's method, ${ }^{20}$ NetCBI, ${ }^{23}$ RLSMDA, ${ }^{30}$ and KATZ. ${ }^{36}$

Using our proposed similarity computation method to measure the similarity of miRNA and disease, LOOCV is implemented on the benchmark dataset; and receiver operating characteristic (ROC) curve and the area under ROC curve (AUC) are adopted to evaluate the predicted performance of NetCF and comparison methods. The four parameters of NetCF are set to $\alpha=0.5, \beta=\mathrm{nm} /(\mathrm{nm}+\mathrm{nd}), n=47$, and $m=33$. Optimal parameters are selected for Liu's method, NetCBI, RLSMDA, and KATZ as described in their literatures. The ROC curves of NetCF and comparison methods are plotted in Fig. 3, and the AUC values are indicated in the legends.

The AUC value of NetCF is 0.8960 , whereas those of Liu's method, NetCBI, RLSMDA, and KATZ are 0.7974, 0.8105, 0.8406, and 0.8315 , respectively. All methods obtained a reliable AUC value when LOOCV is implemented on the benchmark dataset, which proves the rationality of our miRNA and disease similarity measure.

Evidently, NetCF shows better predicted performance compared with Liu's method, NetCBI, RLSMDA, and KATZ.

\subsection{Parameters effects}

The value of $\Delta$ is set to 0.5 according to the suggestion in author's. ${ }^{14}$ The parameter $n$ represents the number of the most similar neighbors of the query miRNA, and $10 \%$ of the number 
Table 2 The top 50 potential prostate cancer-related miRNAs predicted by NetCF, 47 of them were confirmed by dbDEMC and PhenomiR databases

\begin{tabular}{|c|c|c|}
\hline Rank & miRNA & Evidences \\
\hline 1 & hsa-mir-18a & $\begin{array}{l}\text { dbDEMC, } \\
\text { PhenomiR }\end{array}$ \\
\hline 2 & hsa-mir-155 & PhenomiR \\
\hline 3 & hsa-mir-429 & Unconfirmed \\
\hline 4 & hsa-mir-9 & $\begin{array}{l}\text { dbDEMC, } \\
\text { PhenomiR }\end{array}$ \\
\hline 5 & hsa-mir-19b & $\begin{array}{l}\text { dbDEMC, } \\
\text { PhenomiR }\end{array}$ \\
\hline 6 & hsa-mir-19a & $\begin{array}{l}\text { dbDEMC, } \\
\text { PhenomiR }\end{array}$ \\
\hline 7 & hsa-mir-181a & $\begin{array}{l}\text { dbDEMC, } \\
\text { PhenomiR }\end{array}$ \\
\hline 8 & hsa-mir-196a & $\begin{array}{l}\text { dbDEMC, } \\
\text { PhenomiR }\end{array}$ \\
\hline 9 & hsa-mir-29c & $\begin{array}{l}\text { dbDEMC, } \\
\text { PhenomiR }\end{array}$ \\
\hline 10 & hsa-mir-10b & PhenomiR \\
\hline 11 & hsa-mir-138 & PhenomiR \\
\hline 12 & hsa-mir-24 & $\begin{array}{l}\text { dbDEMC, } \\
\text { PhenomiR }\end{array}$ \\
\hline 13 & hsa-mir-7 & $\begin{array}{l}\text { dbDEMC, } \\
\text { PhenomiR }\end{array}$ \\
\hline 14 & hsa-mir-210 & $\begin{array}{l}\text { dbDEMC, } \\
\text { PhenomiR }\end{array}$ \\
\hline 15 & hsa-mir-150 & PhenomiR \\
\hline 16 & hsa-mir-451a & dbDEMC \\
\hline 17 & hsa-let-7e & $\begin{array}{l}\text { dbDEMC, } \\
\text { PhenomiR }\end{array}$ \\
\hline 18 & hsa-mir-30a & $\begin{array}{l}\text { dbDEMC, } \\
\text { PhenomiR }\end{array}$ \\
\hline 19 & hsa-mir-125a & $\begin{array}{l}\text { dbDEMC, } \\
\text { PhenomiR }\end{array}$ \\
\hline 20 & hsa-mir-149 & $\begin{array}{l}\text { dbDEMC, } \\
\text { PhenomiR }\end{array}$ \\
\hline 21 & hsa-mir-103a & dbDEMC \\
\hline 22 & hsa-let-7g & $\begin{array}{l}\text { dbDEMC, } \\
\text { PhenomiR }\end{array}$ \\
\hline 23 & hsa-mir-192 & dbDEMC \\
\hline 24 & hsa-mir-186 & $\begin{array}{l}\text { dbDEMC, } \\
\text { PhenomiR }\end{array}$ \\
\hline 25 & hsa-mir-140 & dbDEMC \\
\hline 26 & hsa-mir-20b & dbDEMC \\
\hline 27 & hsa-mir-302d & PhenomiR \\
\hline 28 & hsa-mir-128 & $\begin{array}{l}\text { dbDEMC, } \\
\text { PhenomiR }\end{array}$ \\
\hline 29 & hsa-mir-328 & $\begin{array}{l}\text { dbDEMC, } \\
\text { PhenomiR }\end{array}$ \\
\hline 30 & hsa-mir-215 & $\begin{array}{l}\text { dbDEMC, } \\
\text { PhenomiR }\end{array}$ \\
\hline 31 & hsa-mir-383 & $\begin{array}{l}\text { dbDEMC, } \\
\text { PhenomiR }\end{array}$ \\
\hline 32 & hsa-mir-26b & $\begin{array}{l}\text { dbDEMC, } \\
\text { PhenomiR }\end{array}$ \\
\hline 33 & hsa-mir-302a & PhenomiR \\
\hline 34 & hsa-let-7f & $\begin{array}{l}\text { dbDEMC, } \\
\text { PhenomiR }\end{array}$ \\
\hline 35 & hsa-mir-181d & dbDEMC \\
\hline 36 & hsa-mir-142 & PhenomiR \\
\hline 37 & hsa-mir-449b & Unconfirmed \\
\hline 38 & hsa-mir-197 & $\begin{array}{l}\text { dbDEMC, } \\
\text { PhenomiR }\end{array}$ \\
\hline
\end{tabular}

Table 2 (Contd.)

\begin{tabular}{|c|c|c|}
\hline Rank & miRNA & Evidences \\
\hline 39 & hsa-mir-10a & $\begin{array}{l}\text { dbDEMC, } \\
\text { PhenomiR }\end{array}$ \\
\hline 40 & hsa-mir-302b & PhenomiR \\
\hline 41 & hsa-mir-615 & dbDEMC \\
\hline 42 & hsa-mir-365a & dbDEMC \\
\hline 43 & hsa-mir-92b & Unconfirmed \\
\hline 44 & hsa-mir-139 & $\begin{array}{l}\text { dbDEMC, } \\
\text { PhenomiR }\end{array}$ \\
\hline 45 & hsa-mir-423 & $\begin{array}{l}\text { dbDEMC, } \\
\text { PhenomiR }\end{array}$ \\
\hline 46 & hsa-mir-212 & $\begin{array}{l}\text { dbDEMC, } \\
\text { PhenomiR }\end{array}$ \\
\hline 47 & hsa-mir-137 & PhenomiR \\
\hline 48 & hsa-mir-181c & $\begin{array}{l}\text { dbDEMC, } \\
\text { PhenomiR }\end{array}$ \\
\hline 49 & hsa-mir-497 & $\begin{array}{l}\text { dbDEMC, } \\
\text { PhenomiR }\end{array}$ \\
\hline 50 & hsa-mir-302c & PhenomiR \\
\hline
\end{tabular}

of miRNAs is empirically set. The parameter $m$ is set in the same way. In this section, we mainly discuss the influence of the weight coefficient $\alpha$ and the integrated parameter $\beta$ on the predictive performance of NetCF. To evaluate the effects of the integrated parameter $\beta$, the other parameters are fixed $(\alpha=0.5$, $\Delta=0.5, n=47$, and $m=33$ ), and the value of parameter $\beta$ changes from 0.1 to 0.9 . Then, according to the optimal $\beta$ value, the effects of parameter $\alpha$ on NetCF are tested in the same way. The results are shown in Fig. 4 . When the parameter $\alpha$ varies from 0.1 to 0.9 , the range of AUCs is 0.8927 to 0.8970 ; and when the parameter $\beta$ varies from 0.1 to 0.9 , the range of AUCs is 0.8732 to 0.8970 . Therefore, NetCF is not sensitive to these two parameters. And for the integrated parameter $\beta$, we do not want to be too focused on miRNA- or disease-similaritybased recommendation score. In the experiment we set $\beta=$ $\mathrm{nm} /(\mathrm{nm}+\mathrm{nd})$, and the other parameters are set to $\alpha=0.5, \Delta=$ $0.5, n=47$, and $m=33$.

\subsection{Case study}

A large number of researchers have indicated that miRNA plays an important role in the development of various forms of cancer from different perspectives. To further evaluate the predicted performance of NetCF for predicting potential disease-related miRNAs, lung and prostate cancers are chosen as case studies. All known associations are used as training set, and recommendation scores are calculated using NetCF. The unknown associations of each disease are ranked according to their recommendation scores, and the top 50 are selected for further validation. The predicted results are verified based on the recently updated $\mathrm{dbDEMC}^{10}$ and PhenomiR ${ }^{12}$ databases. To explore aberrantly expressed miRNAs in different cancers, the dbDEMC database is used as different experimental designs and data sources. To obtain reliable results, we only chose the results of the experimental conditions "cancer $v s$. normal" and the data from TCGA (The Cancer Genome Atlas, https:// 
Table 3 The top 50 potential lung cancer-related miRNAs predicted by NetCF with removed all known lung cancer-miRNA associations, all of them were confirmed based on the dbDEMC and PhenomiR databases

\begin{tabular}{|c|c|}
\hline Rank & miRNA \\
\hline 1 & hsa-mir-16 \\
\hline 2 & hsa-mir-15a \\
\hline 3 & hsa-mir-195 \\
\hline 4 & hsa-mir-141 \\
\hline 5 & hsa-mir-151a \\
\hline 6 & hsa-mir-130a \\
\hline 7 & hsa-mir-302b \\
\hline 8 & hsa-mir-106b \\
\hline 9 & hsa-mir-429 \\
\hline 10 & hsa-mir-296 \\
\hline 11 & hsa-mir-122 \\
\hline 12 & hsa-mir-451a \\
\hline 13 & hsa-mir-99a \\
\hline 14 & hsa-mir-193b \\
\hline 15 & hsa-mir-708 \\
\hline 16 & hsa-mir-378a \\
\hline 17 & hsa-mir-302c \\
\hline 18 & hsa-mir-152 \\
\hline 19 & hsa-mir-625 \\
\hline 20 & hsa-mir-204 \\
\hline 21 & hsa-mir-15b \\
\hline 22 & hsa-mir-149 \\
\hline 23 & hsa-mir-328 \\
\hline 24 & hsa-mir-20b \\
\hline 25 & hsa-mir-129 \\
\hline 26 & hsa-mir-139 \\
\hline 27 & hsa-mir-302a \\
\hline 28 & hsa-mir-194 \\
\hline 29 & hsa-mir-10a \\
\hline 30 & hsa-mir-320a \\
\hline 31 & hsa-mir-449a \\
\hline 32 & hsa-mir-302d \\
\hline 33 & hsa-mir-196b \\
\hline 34 & hsa-mir-148b \\
\hline 35 & hsa-mir-215 \\
\hline 36 & hsa-mir-151b \\
\hline 37 & hsa-mir-99b \\
\hline
\end{tabular}

Table 3 (Contd.)

\begin{tabular}{|c|c|c|}
\hline Rank & miRNA & Evidences \\
\hline 38 & hsa-mir-452 & $\begin{array}{l}\text { dbDEMC, } \\
\text { PhenomiR }\end{array}$ \\
\hline 39 & hsa-mir-367 & PhenomiR \\
\hline 40 & hsa-mir-342 & $\begin{array}{l}\text { dbDEMC, } \\
\text { PhenomiR }\end{array}$ \\
\hline 41 & hsa-mir-373 & PhenomiR \\
\hline 42 & hsa-mir-345 & $\begin{array}{l}\text { dbDEMC, } \\
\text { PhenomiR }\end{array}$ \\
\hline 43 & hsa-mir-449b & PhenomiR \\
\hline 44 & hsa-mir-339 & $\begin{array}{l}\text { dbDEMC, } \\
\text { PhenomiR }\end{array}$ \\
\hline 45 & hsa-mir-425 & $\begin{array}{l}\text { dbDEMC, } \\
\text { PhenomiR }\end{array}$ \\
\hline 46 & hsa-mir-23b & $\begin{array}{l}\text { dbDEMC, } \\
\text { PhenomiR }\end{array}$ \\
\hline 47 & hsa-mir-130b & $\begin{array}{l}\text { dbDEMC, } \\
\text { PhenomiR }\end{array}$ \\
\hline 48 & hsa-mir-211 & PhenomiR \\
\hline 49 & hsa-mir-92b & PhenomiR \\
\hline 50 & hsa-mir-181d & $\begin{array}{l}\text { dbDEMC, } \\
\text { PhenomiR }\end{array}$ \\
\hline
\end{tabular}

cancergenome.nih.gov/). By collecting data from published studies, the PhenomiR database provides information on differentially regulated miRNA expression in diseases and other biological processes. The top 50 potential lung cancer- and prostate cancer-related miRNAs predicted by NetCF and the confirmation by the dbDEMC and PhenomiR databases are listed in Tables 1 and 2.

The high mortality rate of lung cancer makes it the most common cause of cancer-related death in men and second in women. ${ }^{37}$ Many researchers have demonstrated that miRNA dysregulation is associated with lung cancer, and in the benchmark dataset, 128 lung cancer-related miRNAs are verified by biological experiments. Unknown lung cancer-related miRNAs are predicted by NetCF. Among the top 50 predicted miRNAs, 47 of them are confirmed by the dbDEMC and PhenomiR databases; and only 3 miRNAs (hsa-mir-520g, hsa-mir630 and hsa-mir-519c, ranked $41^{\text {st }}, 47^{\text {th }}$ and $49^{\text {th }}$, respectively) are not confirmed. The confirmation of the top 40 predictions is particularly gratifying. Moreover, Cao et al. ${ }^{38}$ reported that hasmir-630 inhibits cell proliferation of lung cancer by targeting cell-cycle kinase 7 (CDC7); and Cha et al. ${ }^{39}$ identified has-mir$519 \mathrm{c}$ as a tumor suppressor involved in lung cancer progression.

Prostate cancer is the most common cancer in males in 84 countries, ${ }^{37}$ occurring more commonly in the developed world.

Biological experiments have demonstrated several important associations between prostate cancer and dysregulation of miRNAs. NetCF is implemented to predict potential prostate cancer-related miRNAs. Of the top 50 predicted miRNAs, 47 are confirmed based on the dbDEMC and PhenomiR databases; and only 3 miRNAs (hsa-mir-429, hsa-mir-449b and hsa-mir$92 \mathrm{~b}$, ranked third, $37^{\text {th }}$ and $43^{\text {rd }}$, respectively) are not found in the two databases. Further literature search demonstrated that hsa-mir-429 inhibits cell proliferation by targeting p27Kip1 in human prostate cancer cells. ${ }^{40}$ 
Table 4 The top 50 potential prostate cancer-related miRNAs predicted by NetCF with removed all known prostate cancer-miRNA associations, 48 of them were confirmed based on the dbDEMC and PhenomiR databases

\begin{tabular}{|c|c|}
\hline Rank & miRNA \\
\hline 1 & hsa-mir-18a \\
\hline 2 & hsa-mir-155 \\
\hline 3 & hsa-mir-19a \\
\hline 4 & hsa-mir-9 \\
\hline 5 & hsa-mir-10b \\
\hline 6 & hsa-mir-210 \\
\hline 7 & hsa-mir-19b \\
\hline 8 & hsa-mir-181a \\
\hline 9 & hsa-mir-7 \\
\hline 10 & hsa-mir-138 \\
\hline 11 & hsa-mir-196a \\
\hline 12 & hsa-mir-24 \\
\hline 13 & hsa-mir-142 \\
\hline 14 & hsa-mir-29c \\
\hline 15 & hsa-mir-30a \\
\hline 16 & hsa-mir-125a \\
\hline 17 & hsa-mir-302b \\
\hline 18 & hsa-mir-199b \\
\hline 19 & hsa-let-7i \\
\hline 20 & hsa-let-7g \\
\hline 21 & hsa-let-7e \\
\hline 22 & hsa-mir-499a \\
\hline 23 & hsa-mir-150 \\
\hline 24 & hsa-mir-429 \\
\hline 25 & hsa-mir-135a \\
\hline 26 & hsa-let-7f \\
\hline 27 & hsa-mir-451a \\
\hline 28 & hsa-mir-192 \\
\hline 29 & hsa-mir-302c \\
\hline 30 & hsa-mir-18b \\
\hline 31 & hsa-mir-139 \\
\hline 32 & hsa-mir-103a \\
\hline 33 & hsa-mir-625 \\
\hline 34 & hsa-mir-140 \\
\hline 35 & hsa-mir-20b \\
\hline 36 & hsa-mir-215 \\
\hline 37 & hsa-mir-128 \\
\hline 38 & hsa-mir-129 \\
\hline
\end{tabular}

Table 4 (Contd.)

\begin{tabular}{|c|c|c|}
\hline Rank & miRNA & Evidences \\
\hline 39 & hsa-mir-137 & PhenomiR \\
\hline 40 & hsa-mir-302a & PhenomiR \\
\hline 41 & hsa-mir-10a & $\begin{array}{l}\text { dbDEMC, } \\
\text { PhenomiR }\end{array}$ \\
\hline 42 & hsa-mir-149 & $\begin{array}{l}\text { dbDEMC, } \\
\text { PhenomiR }\end{array}$ \\
\hline 43 & hsa-mir-26b & $\begin{array}{l}\text { dbDEMC, } \\
\text { PhenomiR }\end{array}$ \\
\hline 44 & hsa-mir-328 & $\begin{array}{l}\text { dbDEMC, } \\
\text { PhenomiR }\end{array}$ \\
\hline 45 & hsa-mir-497 & $\begin{array}{l}\text { dbDEMC, } \\
\text { PhenomiR }\end{array}$ \\
\hline 46 & hsa-mir-30b & $\begin{array}{l}\text { dbDEMC, } \\
\text { PhenomiR }\end{array}$ \\
\hline 47 & hsa-mir-302d & PhenomiR \\
\hline 48 & hsa-mir-542 & dbDEMC \\
\hline 49 & hsa-mir-342 & $\begin{array}{l}\text { dbDEMC, } \\
\text { PhenomiR }\end{array}$ \\
\hline 50 & hsa-mir-338 & $\begin{array}{l}\text { dbDEMC, } \\
\text { PhenomiR }\end{array}$ \\
\hline
\end{tabular}

\subsection{Application of NetCF to predict isolated disease-related miRNAs}

An isolated disease refers to a disease without any known associated miRNA. To further evaluate the predicted performance of NetCF for predicting isolated disease-related miRNAs, the recommended scores of potential associations are calculated by removing all known associations related to predicted disease. In other words, we only use the similarity information and known associations of other diseases to predict isolated disease-related miRNAs. Isolated disease-related miRNAs prediction is implemented for lung and prostate cancers with the top 50 potential miRNAs are listed in Tables 3 and 4, respectively. For lung cancer, all top 50 predicted miRNAs are confirmed based on the dbDEMC and PhenomiR databases. For prostate cancer, only 2 miRNAs (hsa-mir-499a and hsa-mir-429, ranked $22^{\text {nd }}$ and $24^{\text {th }}$ ) of top 50 predicted results are not found in the two databases; and hsa-mir- 429 is confirmed to be associated with prostate cancer by recently published literature. ${ }^{40}$ Acording to the above description, NetCF exhibits good performance for predicting potential miRNA-disease associations and isolated disease-related miRNAs.

\section{Conclusions}

Accumulative evidence has indicated that miRNAs play important roles in the pathogenesis and development of many complex diseases. The identification of novel disease-associated miRNAs is an important task in biomedical research, and it is beneficial for an in-depth understanding of disease mechanisms at the miRNA level. As a useful supplement to experimental studies, using computational approaches to identify disease-related miRNAs is beneficial in detecting the functions of miRNA biomarker for disease diagnosis, treatment, and prevention. 
In this work, we develop a new method for measuring miRNA and disease similarities based on normalized mutual information. This method combines disease associated genes and disease DAG graphs to calculate disease similarity; and it calculates miRNA similarity based on miRNA-mRNA interactions. Given that no known association information is used in the similarity computation process, LOOCV does not overestimate the predicted performance. We then proposed NetCF for predicting new miRNA-disease associations by integrating miRNA and disease similarities with known miRNA-disease associations. Reliable AUC values for all comparable methods demonstrated that our proposed similarity computation method is reasonable and feasible. The AUC value of NetCF is superior to the other comparable methods, which indicates that NetCF has reliable predicted performance. Case studies further demonstrated the good predicted performance of NetCF for predicting potential and isolated disease-related miRNAs.

Even with the favorable results obtained using our method, this study reveals certain limitations. First, miRNA pair similarity is calculated based on the known common target genes because known miRNA-mRNA are scarce; thus, the similarities of many miRNA pairs are 0 . The problem will be addressed considering that miRNA-target genes are increasingly recognized. In our future work, we will integrate more miRNA-related data to further improve the miRNA similarity measure. Second, future work should consider parameter optimization. For example, for the numbers of miRNA's neighbors and disease's neighbors, we separately choose $10 \%$ of the numbers of miRNAs and diseases based on experiments. This selection works well in our dataset, but not necessarily for other datasets.

\section{Conflicts of interest}

There are no conflicts to declare.

\section{Acknowledgements}

This work is supported by the Program for National Nature Science Foundation of China (61672214, 61672223, 61572178, 61370171 and 61472127).

\section{References}

1 S. Chatterjee and H. Grosshans, Nature, 2009, 461, 546-549. 2 J. T. Mendell and E. N. Olson, Cell, 2012, 148, 1172-1187.

3 E. M. Small and E. N. Olson, Nature, 2011, 469, 336-342.

4 W. Shi, J. Du, Y. Qi, G. Liang, T. Wang, S. Li, S. Xie, B. Zeshan and Z. Xiao, J. Psychiatr. Res., 2012, 46, 198-204.

5 E. N. Olson, Sci. Transl. Med., 2014, 6(239), 239 ps3.

6 Y. W. Kong, D. Ferland-McCollough, T. J. Jackson and M. Bushell, Lancet Oncol., 2012, 13, e249-258.

7 T. T. Manavalan, Y. Teng, S. N. Appana, S. Datta, T. S. Kalbfleisch, Y. Li and C. M. Klinge, Cancer Lett., 2011, 313, 26-43.

8 Y. Li, C. Qiu, J. Tu, B. Geng, J. Yang, T. Jiang and Q. Cui, Nucleic Acids Res., 2014, 42, D1070-D1074.
9 Q. Jiang, Y. Wang, Y. Hao, L. Juan, M. Teng, X. Zhang, M. Li, G. Wang and Y. Liu, Nucleic Acids Res., 2009, 37, D98-D104. 10 Z. Yang, L. Wu, A. Wang, W. Tang, Y. Zhao, H. Zhao and A. E. Teschendorff, Nucleic Acids Res., 2017, 45, D812-D818. 11 Z. Yang, F. Ren, C. Liu, S. He, G. Sun, Q. Gao, L. Yao, Y. Zhang, R. Miao, Y. Cao, Y. Zhao, Y. Zhong and H. Zhao, BMC Genomics, 2010, 11(4), S5.

12 A. Ruepp, A. Kowarsch, D. Schmidl, F. Buggenthin, B. Brauner, I. Dunger, G. Fobo, G. Frishman, C. Montrone and F. J. Theis, Genome Biol., 2010, 11, R6.

13 X. X. Zeng, X. Zhang and Q. Zou, Briefings Bioinf., 2016, 17, 193-203.

14 D. Wang, J. Wang, M. Lu, F. Song and Q. Cui, Bioinformatics, 2010, 26, 1644-1650.

15 J. Freudenberg and P. Propping, Bioinformatics, 2002, 18(2), S110-S115.

16 M. A. van Driel, J. Bruggeman, G. Vriend, H. G. Brunner and J. A. Leunissen, Eur. J. Hum. Genet., 2006, 14, 535-542.

17 Q. Zou, J. Li, L. Song, X. Zeng and G. Wang, Briefings Funct. Genomics, 2016, 15, 55-64.

18 Q. Jiang, Y. Hao, G. Wang, et al., BMC Syst. Biol., 2010, 4(1), S2.

19 Q. Jiang, G. Wang and Y. Wang, An approach for prioritizing disease-related microRNAs based on genomic data integration, Biomedical Engineering and Informatics (BMEI), 2010 3rd International Conference On. IEEE, 2010, vol. 6, pp. 2270-2274.

20 Y. Liu, X. Zeng, Z. He and Q. Zou, IEEE/ACM Trans. Comput. Biol. Bioinf., 2016, DOI: 10.1109/TCBB.2016.2550432.

21 X. Chen, M. X. Liu and G. Y. Yan, Mol. BioSyst., 2012, 8, 27922798.

22 H. Shi, J. Xu, G. Zhang, et al., BMC Syst. Biol., 2013, 7(1), 101.

23 H. Chen and Z. Zhang, BMC Med. Genomics, 2013, 6, 12.

24 P. Xuan, K. Han, M. Guo, et al., PloS one, 2013, 8(8), e70204.

25 X. M. Zhao, K. Q. Liu, G. H. Zhu, F. He, B. Duval, J. M. Richer, D. S. Huang, C. J. Jiang, J. K. Hao and L. N. Chen, Bioinformatics, 2015, 31, 1226-1234.

26 G. M. Qin, R. Y. Li and X. M. Zhao, IEEE/ACM Trans. Comput. Biol. Bioinf., 2016, 13, 1027-1035.

27 Q. Jiang, G. Wang, S. Jin, Y. Li and Y. Wang, International journal of data mining and bioinformatics, 2013, 8, 282-293.

28 X. X. Zeng, X. Zhang, Y. L. Liao and L. Q. Pan, Biochim. Biophys. Acta, Gen. Subj., 2016, 1860, 2735-2739.

29 C. Shi, X. N. Kong, Y. Huang, P. S. Yu and B. Wu, IEEE Transactions on Knowledge and Data Engineering, 2014, 26, 2479-2492.

30 X. Chen and G. Y. Yan, Sci. Rep., 2014, 4, DOI: 10.1038/ srep05501.

31 J. Pinero, N. Queralt-Rosinach, A. Bravo, et al., Database, 2015, 2015, DOI: 10.1093/database/bav028.

32 S. D. Hsu, Y. T. Tseng, S. Shrestha, Y. L. Lin, A. Khaleel, C. H. Chou, C. F. Chu, H. Y. Huang, C. M. Lin, S. Y. Ho, T. Y. Jian, F. M. Lin, T. H. Chang, S. L. Weng, K. W. Liao, I. E. Liao, C. C. Liu and H. D. Huang, Nucleic Acids Res., 2014, 42, D78-D85.

33 S. D. Hsu, F. M. Lin, W. Y. Wu, C. Liang, W. C. Huang, W. L. Chan, W. T. Tsai, G. Z. Chen, C. J. Lee, C. M. Chiu, 
C. H. Chien, M. C. Wu, C. Y. Huang, A. P. Tsou and H. D. Huang, Nucleic Acids Res., 2011, 39, D163-D169.

34 F. Ricci, L. Rokach and B. Shapira, Introduction to recommender systems handbook, Springer, 2011.

35 Y. Zhou, B. Song and H. T. Zheng, Exploiting Latent Relations Between Users and Items for Collaborative Filtering, International Conference on Neural Information Processing, Springer, Cham, 2015, pp. 365-374.

36 Q. Zou, J. Li, Q. Hong, et al., BioMed Res. Int., 2015, 2015, DOI: $10.1155 / 2015 / 810514$.
37 S. McGuire, Adv. Nutr., 2016, 7, 418-419.

38 J. X. Cao, Y. Lu, J. J. Qi, G. S. An, Z. B. Mao, H. T. Jia, S. Y. Li and J. H. Ni, Cell Death Discovery, 2014, 5, e1426.

39 S. T. Cha, P. S. Chen, G. Johansson, C. Y. Chu, M. Y. Wang, Y. M. Jeng, S. L. Yu, J. S. Chen, K. J. Chang, S. H. Jee, C. T. Tan, M. T. Lin and M. L. Kuo, Cancer Res., 2010, 70, 2675-2685.

40 Y. Ouyang, P. Gao, B. Zhu, X. Chen, F. Lin, X. Wang, J. Wei and H. Zhang, Mol. Med. Rep., 2015, 11, 1435-1441. 